\title{
Sindrome dos Ovários Policísticos: Avaliação Dopplerfluxométrica
}

\author{
Polycystic Ovary Syndrome: Doppler Flow Measurement Evaluation \\ Ricardo Vasconcellos Bruno, Marco Antonio Pessanha Lourenço \\ Marcio Augusto Pinto de Avila
}

\begin{abstract}
RESUMO
Objetivo: avaliar a eficácia do Doppler colorido como método diagnóstico na síndrome dos ovários policísticos (SOP), mediante as variações do fluxo sangüineo no estroma ovariano, nas artérias uterinas e no tecido subendometrial.

Métodos: foram selecionadas trinta pacientes distribuidas em dois grupos: quinze com amenorréia ou oligomenorréia, hirsutismo (indice de Ferriman e Gallwey >8), indice de massa corporal IMC maior que $25 \mathrm{~kg} / \mathrm{m}^{2}$ e exame ecográfico que identificou estroma aumentado, hiperecogênico e policistose ovariana (grupo de estudo), e número idêntico de pacientes com ciclos menstruais normais, sem sinais de hirsutismo e ultra-sonografia normal (grupo controle). Com o uso da dopplerfluxometria transvaginal foram medidas a velocidade de pico sistólico ou velocidade máxima $\left(V_{\text {máx }}\right)$, indice de pulsatilidade (IP) e resistência dos vasos do estroma ovariano, da artéria uterina e do subendométrio.

Resultados: a dopplervelocimetria mostrou $V_{\text {máx }}$ significativamente maior no estroma ovariano das pacientes com $S O P(12,2 \mathrm{~cm} / \mathrm{s})(p \leq 0,0004)$ em relação ao grupo controle $(8,5 \mathrm{~cm} / \mathrm{s}) . O$ IP da artéria uterina também se revelou muito superior no grupo com $S O P(3,3 \mathrm{~cm} / \mathrm{s}) \mathrm{em}$ relação ao controle $(2,7 \mathrm{~cm} / \mathrm{s})$. Outros parâmetros da dopplervelocimetria não evidenciaram diferença significativa. Ao estabelecermos um cutoff (ponto de corte) $=9 \mathrm{~cm} / \mathrm{s}$ na amostragem para a $V_{\text {máx }}$ obtivemos os percentuais de 95,2 para sensibilidade, 80,0 para especificidade $e$ de 83,3 para o valor preditivo positivo e 94,1 para o negativo. Com referência ao IP para um cutoff $=2.35$ os testes revelaram: sensibilidade de $81,0 \%$, especificidade de $50,0 \%$, valor preditivo positivo de 63,0\% e negativo de $71,4 \%$.

Conclusão: a dopplervelocimetria pode constituir subsidio a ser incorporado à investigação clínica e ultra-sonográfica no tocante ao diagnóstico da sindrome dos ovários policísticos.
\end{abstract}

PALAVRAS-CHAVE: Síndrome dos ovários policísticos. Hirsutismo. Dopplervelocimetria.

\section{Introdução}

A síndrome dos ovários policísticos (SOP) é uma doença plural, multifacetada, que incide em mulheres em idade reprodutiva e suas interações clínicas e bioquímicas ensejam, atualmente, interesse de várias disciplinas.

O ovário, interpretado como sítio originário da SOP, propicia uma diversificação de alterações hormonais influenciadas pela resistência insulínica, que conduz à suspeita de diabetes tipo 2. As alterações do metabolismo lipídico favorecem

Instituto de Ginecologia da Universidade Federal do Rio de Janeiro (UFRJ)

Correspondência:

Ricardo V. Bruno

Rua Conselheiro Zenha, 19/806 - Tijuca

20550-090 - Rio de Janeiro - RJ o risco de doença cardiovascular. Nesse particular, o conceito de síndrome metabólica diz respeito ao vínculo entre a hiperglicemia, a intolerância à glicose, a hipertensão e as alterações do metabolismo lipídico ${ }^{1}$.

A sindrome teve como principal parâmetro diagnóstico a relação hormônio luteinizante (LH)/ hormônio folículo-estimulante (FSH). Esta relação foi determinante para a caracterização da doença quando se apresentava superior a três, evidenciando o aumento expressivo do $\mathrm{LH}$ em detrimento do FSH. Posteriormente Ehrmann et al. ${ }^{2}$ utilizaram a relação maior que dois, como parâmetro de interpretação da síndrome. A avaliação do $\mathrm{LH}$ isoladamente ganhou destaque nos estudos observacionais de Zaidi et al. ${ }^{3}$, que caracterizavam a sindrome quando os niveis se apresentavam acima de $10 \mathrm{U} / \mathrm{L}$, embora Givens 
et al. ${ }^{4}$ contestassem com dados coletados em pacientes com SOP que apresentavam niveis de LH normais. Até os próprios niveis de androgênios como a androstenediona e a testosterona podem apresentar-se modestamente aumentados ou normais ${ }^{5}$. Ainda devemos considerar que os niveis hormonais podem sofrer ação do índice de massa corporal (IMC), do estresse, do fumo, do dia do ciclo e, principalmente, vale salientar, estão constantemente em estado de transformação metabólica ${ }^{6}$.

A ultra-sonografia surgiu como método auxiliar de excelência para o diagnóstico da SOP devido à perfeita visualização da morfologia ovariana. Crowley et al. ${ }^{7}$ definiram critérios para o padrão de imagem no diagnóstico da SOP como: número, volume e diâmetro dos microcistos. O surgimento de novas técnicas de imagem como a ultra-sonografia transvaginal e o Doppler colorido favoreceu a busca por mais subsídios para o diagnóstico da SOP. Battaglia et al. ${ }^{8}$ aferiram e constataram que as variações do fluxo sangüíneo ovariano e uterino estavam associadas aos parâmetros clínicos, ultra-sonográficos e endócrinos típicos da SOP. Estes autores avaliaram 32 pacientes portadoras de SOP e compararam-nas a 18 mulheres sem a doença, dentro dos seus critérios de inclusão. Pela análise dopplerfluxométrica as mulheres com SOP apresentaram indice de pulsatilidade (IP) das artérias uterinas com significativo aumento em relação às pacientes de controle e o índice de resistência (IR) do estroma ovariano foi significativamente menor no grupo com SOP, demonstrando a baixa resistência da vascularização intra-ovariana em presença da doença.

Zaidi et al. ${ }^{3}$ confirmaram as alterações vasculares pelo estudo dopplerfluxométrico, quando estudaram as variações do fluxo sangüíneo de mulheres em acompanhamento para fertilização in vitro, que evidenciavam esterilidade por SOP. Os autores constataram o aumento da velocidade máxima $\left(\mathrm{V}_{\text {máx }}\right)$ do estroma ovariano nas pacientes com SOP, quando as compararam com mulheres fertéis.

Outros dois autores, Aleem e Predanic ${ }^{9}$ também comprovaram a eficácia do Doppler na SOP, em estudo comparativo entre mulheres portadoras da sindrome e normais, notando uma correlação positiva entre o aumento dos niveis de $\mathrm{LH}$ séricos e da velocidade do fluxo sangüíneo no estroma ovariano das pacientes com SOP. O estudo encontrou um aumento da $V_{\text {máx }}$ no estroma ovariano, bem como nas artérias uterinas, além de discreta tendência à diminuição dos IP e IR.

Devemos considerar que o ovário apresenta uma circulação sangüínea discreta no início do ciclo menstrual, e que aumenta com o desenvolver do folículo até atingir seu ponto máximo no período ovulatório e posteriormente com a formação do corpo lúteo. Com base nesses dados, objetivamos demonstrar as alterações dos fluxos vasculares no início do ciclo menstrual, nas pacientes portadoras de SOP, segundo o estudo dopplerfluxométrico da circulação sangüínea intraovariana, uterina e do subendométrio.

O presente estudo visa avaliar a velocimetria Doppler em pacientes com SOP, comparandoas com mulheres de ciclos normais, sem sinais de androgenização e com ultra-sonografia completamente normal.

\section{Pacientes e Métodos}

Trata-se de estudo prospectivo aprovado pela Comissão de Ética em pesquisa do Instituto de Ginecologia da Universidade Federal do Rio de Janeiro - UFRJ. Foram selecionadas quinze pacientes (grupo de estudo) no ambulatório de Ginecologia Endócrina no período entre março de 1999 e maio de 2000. Todas eram portadoras de SOP com média de idade 27,2 $\pm 4,6$ anos. Dez pacientes estavam em amenorréia (ausência de menstruação por mais de 6 meses) e cinco em oligomenorréia (intervalo maior que 35 dias). Todas apresentavam índice de Ferriman e Gallwey ${ }^{10}$ maior que 8. O IMC apresentou mediana de 29,7 $\mathrm{kg} / \mathrm{m}^{2}$. A medida da cintura apresentou média de $99,1 \pm 11,3 \mathrm{~cm}$ no grupo com SOP. A média dos valores de $\mathrm{LH}$ foi de 8,4 $\pm 6,2 \mathrm{U} / 1$ e a relação $\mathrm{LH} / \mathrm{FSH}$ foi de $1,8 \pm 0,9$ no grupo de estudo. Todas apresentavam acne e estavam em acompanhamento por infertilidade. Foram excluídas pacientes com sindrome de Cushing, tiroidopatias e tumores produtores de prolactina ou androgênio. À ultrasonografia, o volume ovariano do grupo de estudo foi em média de $12,6 \pm 3,3 \mathrm{~cm}^{3}$ (ovário direito) e $12,4 \pm 3,3 \mathrm{~cm}^{3}$ (ovário esquerdo), caracterizando o marcado aumento do volume ovariano na SOP, devido ao desenvolvimento do estroma ovariano (Figura 1). O número de cistos com diâmetro entre $5 \mathrm{~mm}$ e $10 \mathrm{~mm}$, foi em média $13,8 \pm 3,3$ no ovário direito e $12,6 \pm 3,1$ no ovário esquerdo, confirmando a micropolicistose da síndrome.

Quinze pacientes com média de idade de $29,3 \pm 5,7$ anos, ciclos regulares, IMC com mediana de $24 \mathrm{~kg} / \mathrm{m}^{2}$, cintura com média de $87,9 \pm 11,5$ $\mathrm{cm}$, isentas de hirsutismo e que não haviam recebido tratamento hormonal no prazo de 3 meses antes do início da investigação constituíram o grupo controle. Os niveis de LH do grupo controle foram em média 4,3 $\pm 3,9$ U/L e a relação $\mathrm{LH} / \mathrm{FSH}$ 


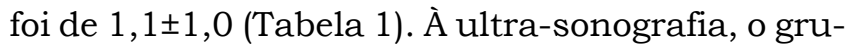
po controle apresentou volume ovariano dentro dos parâmetros considerados para a normalidade com médias de $7,6 \pm 3,6 \mathrm{~cm}^{3}$ (ovário direito) e $7,5 \pm 2,8$ $\mathrm{cm}^{3}$ (ovário esquerdo).

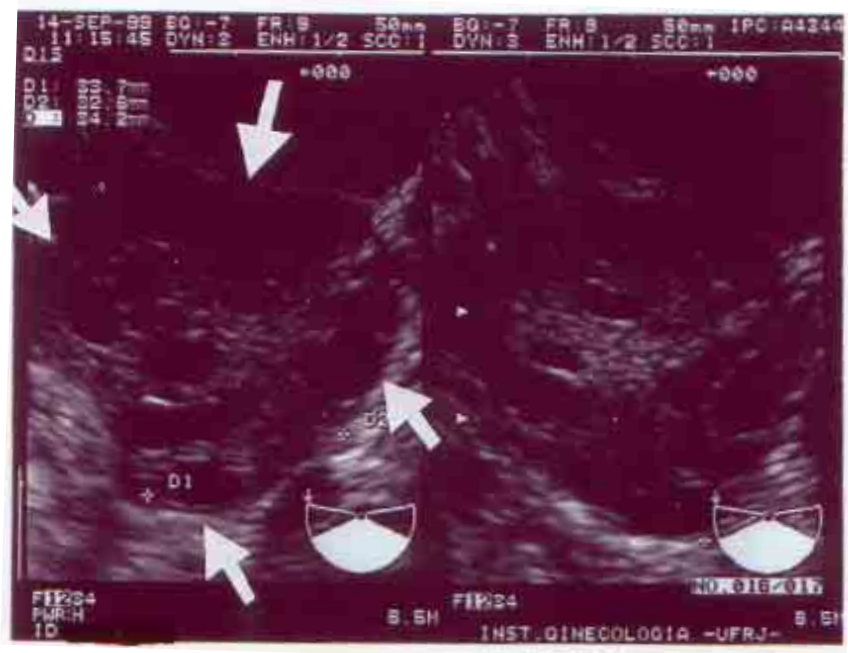

Figura 1 - Ultra-sonografia mostrando ovário aumentado de volume, evidenciando desenvolvimento do estroma e os microcistos.

Tabela 1 - Características clínicas e hormonais dos grupos com síndrome dos ovários policísticos (SOP) e controle e comparação entre ambos por meio do valor de significância estatística $p$.

\begin{tabular}{lccc}
\hline & $\begin{array}{c}\text { Grupo } \\
\text { soP }\end{array}$ & $\begin{array}{c}\text { Grupo } \\
\text { controle }\end{array}$ & $\begin{array}{c}\text { Valor de } \\
\mathbf{p}\end{array}$ \\
\hline Idade & $27,2 \pm 4,6$ & $29,3 \pm 5,7$ & $\mathrm{NSA}$ \\
Cintura (cm) & $99,1 \pm 11,3$ & $87,9 \pm 11,5$ & $0,011^{*}$ \\
LH (U/L) & $8,4 \pm 6,2$ & $4,3 \pm 3,9$ & $0,006^{*}$ \\
FSH (U/L) & $4,4 \pm 1,2$ & $4,3 \pm 2,3$ & $0,39 \mathrm{NS}$ \\
LH/FSH & $1,8 \pm 0,9$ & $1,1 \pm 1,0$ & $0,008^{*}$ \\
Testosterona (ng/dL) & $53,6 \pm 27,0$ & $26,1 \pm 12,3$ & $0,004^{*}$ \\
\hline *D
\end{tabular}

As trinta pacientes foram submetidas à coleta de sangue entre 9:00 e 9:30h, para as dosagens de: LH, FSH, relação LH/FSH e testosterona. Utilizou-se o processo de quimiluminescência ACS: 180 da Ciba-Corning Diagnostics para todos os hormônios. Após a coleta do sangue foi efetuada a ultra-sonografia precedida de micção espontânea, para que não ocorressem alterações no resultado do Doppler ${ }^{11}$. O exame foi realizado na fase proliferativa inicial (3--7ㅇ dia) no período da manhã, de modo a evitarem-se as variações circadianas do fluxo na artéria uterina ${ }^{12}$. Às mulheres em amenorréia foi administrado acetato de medroxiprogesterona, na dose de $10 \mathrm{mg}$ por dia durante 7 dias e após a menstruação seguiam o protocolo normal de exames na fase inicial. Foi utilizado aparelho Hitachi EUB 515 A com transdutor de $6,5 \mathrm{MHz}$ e filtro de $50 \mathrm{WF}$, acoplado ao transdutor, preservativo não-lubrificado e gel Hi-Jelly Hitachi. Foi avaliado o volume ovariano segundo a fórmula $\mathrm{V}=\mathrm{p} / 6^{\prime} \mathrm{D} 11^{\prime} \mathrm{D} 2^{\prime} \mathrm{D} 3$, sendo D1 o diâmetro longitudinal, D2 o ântero-posterior e D3 o transverso.

Procedeu-se à aferição da velocidade de fluxo sangüíneo no pico máximo da sístole, constituindo a $V_{\text {máx }} \mathrm{em} \mathrm{cm} / \mathrm{s}$, e calcularam-se o IP e o $\mathrm{IR}^{13}$. Os três parâmetros foram avaliados nos vasos intraovarianos, nas artérias uterinas e no subendométrio. O ângulo de insonação foi adaptado de modo a obter o máximo de intensidade de cor e, portanto, melhor desempenho do Doppler na interpretação da velocidade do fluxo sangüíneo dos vasos em questão. Todos os exames foram realizados pelo mesmo examinador.

A análise estatística foi realizada pelo teste $t$ de Student ou pelo teste não-paramétrico de Mann-Whitney, para comparação entre os dois grupos (SOP e controle) das diferentes variáveis numéricas avaliadas $\left(\mathrm{V}_{\text {máx }}\right.$ de pico sistólico, IP e IR). O critério de determinação de significância foi o nivel de $5 \%$, ou seja, para valor de p menor ou igual a 0,05, atribui-se significância estatística. Para o parâmetro $\left(\mathrm{V}_{\text {maxx }}\right)$ foi estabelecido um cutoff $=9 \mathrm{~cm} / \mathrm{s}$, para se chegar aos percentuais de sensibilidade, especificidade, teste preditivo positivo e teste preditivo negativo. O cutoff foi necessário por não existir parâmetro de normalidade para indices dopplerfluxométricos na literatura.

\section{Resultados}

Os resultados apresentados são valores médios das aferições dos fluxos sangüineos entre os ovários e entre as artérias uterinas, devido ao fato de todas as pacientes do grupo de estudo serem portadoras de policistose ovariana bilateralmente. No grupo controle os mesmos padrões de medida foram adotados.

O estudo hemodinâmico (Tabela 2) demonstrou um significante aumento da $\mathrm{V}_{\text {máx }}$ no estroma ovariano do grupo com SOP com média de $12,1 \pm 1,8$ $\mathrm{cm} / \mathrm{s}$ e mediana de $12,3 \mathrm{~cm} / \mathrm{s}$. O grupo controle evidenciou $\mathrm{V}_{\text {máx }}$ com média de $8,0 \pm 3,4 \mathrm{~cm} / \mathrm{s}$ e mediana de $7,2 \mathrm{~cm} / \mathrm{s}$. A análise estatística mostrou um p de 0,0004, quando comparados os grupos. Os vasos intra-ovarianos nas pacientes com SOP foram facilmente evidenciados pela dopplerfluxometria colorida, confirmando o aumento da trama vascular nas pacientes com SOP (Figura 2), diferentemente do que ocorre nas pacientes com 
os ovários normais. No estroma o IP apresentou média de 0,8 $\pm 0,1$ e mediana também de 0,8 . O IR apresentou média de $0,5 \pm 0,0$ e mediana de 0,5 . No grupo controle o IP no estroma ovariano foi de $0,9 \pm 0,4$ em média e a mediana de 0,7 . O IR evidenciou média de 0,5 $\pm 0,1$ e mediana de 0,5 . Não houve diferença significante entre os grupos quanto ao IP e ao IR.

Tabela 2 - Médias para velocidade máxima $\left(\mathrm{V}_{\text {max }}\right)$, índice de pulsatilidade $(\mathrm{IP})$ e índice de resistência (IR) referentes aos vasos intraovarianos e artéria uterina na comparação entre os grupos.

\begin{tabular}{|c|c|c|c|}
\hline & $\begin{array}{c}\text { Grupo } \\
\text { SOP }\end{array}$ & $\begin{array}{c}\text { Grupo } \\
\text { controle }\end{array}$ & $\begin{array}{c}\text { Grupo } \\
\text { controle }\end{array}$ \\
\hline \multicolumn{4}{|c|}{ Estroma ovariano } \\
\hline $\mathrm{V}_{\text {máx }}(\mathrm{cm} / \mathrm{s})$ & $12,2 \pm 1,8$ & $8,0 \pm 3,4$ & $8,0 \pm 3,4$ \\
\hline \multicolumn{4}{|c|}{ Estroma ovariano } \\
\hline IP & $0,8 \pm 0,1$ & $0,9 \pm 0,5$ & $0,9 \pm 0,5$ \\
\hline \multicolumn{4}{|c|}{ Estroma ovariano } \\
\hline IR & $0,5 \pm 0,0$ & $0,5 \pm 0,1$ & $0,5 \pm 0,1$ \\
\hline \multicolumn{4}{|l|}{ Artéria uterina } \\
\hline $\mathrm{V}_{\text {máx }}(\mathrm{cm} / \mathrm{s})$ & $27,1 \pm 8,1$ & $29,0 \pm 12,3$ & $29,0 \pm 12,3$ \\
\hline \multicolumn{4}{|l|}{ Artéria uterina } \\
\hline IP & $3,3 \pm 1,0$ & $2,7 \pm 0,9$ & $2,7 \pm 0,9$ \\
\hline \multicolumn{4}{|l|}{ Artéria uterina } \\
\hline IR & $0,8 \pm 0,1$ & $0,8 \pm 0,0$ & $0,8 \pm 0,0$ \\
\hline
\end{tabular}

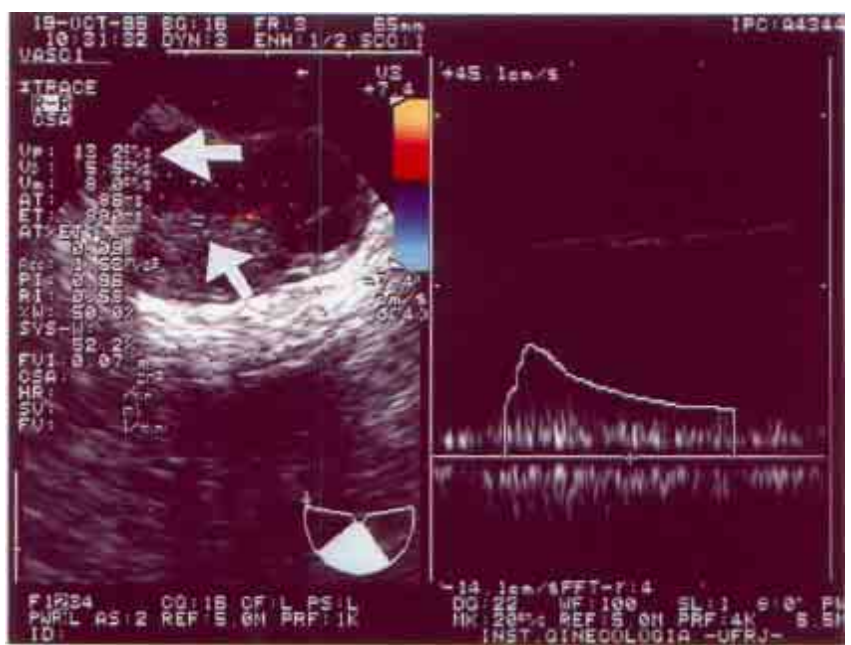

Figura 2 - Doppler do estroma ovariano; notar o aumento da vascularização com os feixes coloridos e o valor da velocidade máxima representado na foto por $\mathrm{Vp}$.

$\mathrm{Na}$ artéria uterina a $\mathrm{V}_{\text {máx }}$ do grupo com SOP apresentou média de $27,0 \pm 8,0 \mathrm{~cm} / \mathrm{s}$ e mediana de $29,5 \mathrm{~cm} / \mathrm{s}$ e no grupo controle a $V_{\text {máx }}$ foi de $29,0 \pm 12,2 \mathrm{~cm} / \mathrm{s}$ (média) e $24,9 \mathrm{~cm} / \mathrm{s}$ (mediana), sem diferença significante. O IP da artéria uterina do grupo com SOP foi de 3,3 $\pm 1,0$ (média) e 3,0 (mediana), já no grupo controle o IP foi de $2,7 \pm 0,9$ (média) e 2,3 (mediana), demonstrando diferença $(p=$ $0,034)$. O IR do grupo portador de SOP foi de $0,8 \pm 0,1$ (média) e 0,8 (mediana), e o grupo controle apresentou média de $0,8 \pm 0,0$ e mediana de 0,8 . No tecido subendometrial a $\mathrm{V}_{\text {máx }}$, IP e IR não revelaram valor estatístico de significância.

\section{Discussão}

As pacientes portadoras da doença policística ovariana apresentaram alguns fatores de risco importantes para doença cardiovascular: IMC aproximadamente $30 \mathrm{~kg} / \mathrm{m}^{2}$, compativel com obesidade, e cintura de aproximadamente $100 \mathrm{~cm}$, excedendo o limite aceitável para adiposidade abdominal. Estes fatores de risco confirmam a preocupação atual da associação da SOP com a doença coronária ${ }^{14}$, justificando a busca por novos subsídios que possam elucidar melhor a fisiopatologia da doença.

Nosso estudo contempla mudanças fundamentais que ocorrem na vascularização intra-ovariana, que sugerem ser o estroma ovariano sítio original da $\mathrm{SOP}^{3,8,9,12,15,16}$.

As alterações vasculares demonstradas pelo nosso estudo na fase inicial do ciclo menstrual (3ํ-7ํ dia) não são vistas no ovário normal. Esse aumento da trama vascular confirmado pela ultrasonografia e o aumento do fluxo sangüíneo confirmado pelo Doppler sugerem a presença de angiogênese ovariana nas pacientes portadoras de SOP. Os niveis de LH mostraram-se aumentados, mas com grande variabilidade, fato este decorrente da alta pulsatilidade do hormônio, como observou Dewailly ${ }^{5}$. A testosterona apresentou níveis com diferença $(p=0,004)$, muito superiores no grupo com SOP em relação ao controle, confirmando o perfil androgênico da patologia ${ }^{17}$.

Os niveis de LH aumentados na sindrome, apesar de sua variabilidade, indicam a sua provável influência na neoangiogênese do estroma ovariano. O mecanismo que proporcionaria este efeito ainda permanece em estudo por Battaglia et al. ${ }^{8}$, que analisam o mecanismo de estimulação catecolaminérgica, associado a uma mediação pelos leucócitos e uma ativação pelas citocinas.

Agrawal et al. ${ }^{18}$ estudaram o fator de crescimento endotelial vascular, cujos niveis aumentados foram associados ao aumento do fluxo sangüíneo no estroma ovariano nas portadoras de SOP, e o LH seria o mediador determinante para este aumento. Os autores ainda chamam a atenção para os riscos da hiperestimulação ovariana 
em pacientes que se encontram em tratamento por intermédio da fertilização in vitro, visto que o aumento do fluxo sangüineo intra-ovariano, na presença de drogas para estimulação da ovulação, poderia conduzir à síndrome de hiperestimulação e, como conseqüência, a cistos ovarianos volumosos.

Engmann et al. ${ }^{19}$ avaliaram a eficácia da velocimetria Doppler no acompanhamento da fertilização in vitro. O trabalho revelou a diminuição da velocidade de fluxo do estroma ovariano em pacientes com SOP, desde que submetidas à supressão ovariana, previamente à estimulação para captação de óvulos, portanto sem os riscos da hiperestimulação, o que confirmou a excelência da velocimetria Doppler no acompanhamento dessas pacientes.

A experiência aqui referida é similar à relatada por Zaidi et al. ${ }^{3}$, que encontraram na $\mathrm{V}_{\text {máx }} \mathrm{O}$ parâmetro que melhor reflete o processo de neoangiogênese do estroma ovariano, embora não tenham sido evidenciadas alterações na impedância (IP e IR) no estroma. Em contrapartida, Battaglia et al. ${ }^{8}$ confirmaram o aumento do IP nas artérias uterinas e diminuição do IR nas artérias intra-ovarianas ${ }^{8,20,21}$. Nossa experiência concorda com Battaglia et $a 1 .^{8}$, pois demonstra a alteração do IP da artéria uterina, sem, no entanto, revelar qualquer modificação no IR, mas devemos ressaltar que as três pesquisas concordam quanto às profundas modificações vasculares a que são submetidas as pacientes portadoras de SOP.

À medida que estudos mais abrangentes longitudinais sejam desenvolvidos, infere-se a possibilidade de o emprego da ultra-sonografia Doppler interagir com os dados clínicos e bioquímicos. Nesse particular, o conceito hemodinâmico (dopplerfluxometria) como subsídio diagnóstico e prognóstico contribui para a elucidação dos intricados mecanismos que regem a fisiopatologia da SOP.

\section{SUMMARY}

Purpose: to evaluate the effectiveness of color Doppler as a diagnosis method for polycystic ovary syndrome (PCOS) through blood flow variations in the ovarian stroma, in the uterine arteries and in the subendometrial tissue.

Methods: thirty patients divided into two groups were selected: fifteen patients with amenorrhea or oligomenorrhea, hirsutism (Ferriman and Gallwey score $>8$ ), body mass index $>25 \mathrm{~kg} / \mathrm{m}^{2}$ and echographic examination identifying increased hyperechogenic stromal and ovarian polycystosis (study group), and an identical number of patients presenting normal menstrual cycles, with no signs of hirsutism and with normal ultrasonography (control group). Transvaginal Doppler flowmetry measured systolic peak velocity or maximal velocity $\left(V_{\max }\right)$ pulsatility index $(P I)$ and resistance of ovarian stromal vessels, uterine arteries and subendometrial layer.

Results: Doppler velocimetry showed significantly higher $V_{\max }$ layer $(p \leq 0,0004)$ in the ovarian stromal of patients with PCOS $(12.2 \mathrm{~cm} / \mathrm{s})$ when compared to the control group $(8.05 \mathrm{~cm} /$ s); the uterine artery PI was also higher in the PCOS group $(3.3 \mathrm{~cm} / \mathrm{s})$ versus the control group $(2.7 \mathrm{~cm} / \mathrm{s})$; other Doppler velocimetry parameters did not show significant differences. As we established a cutoff $=9 \mathrm{~cm} / \mathrm{s}$ for the sample for $V_{\max }$, we obtained the percentages of 95.2 for sensitivity, 80.0 for specificity, 83.3 for positive predictive value and 94.1 for negative predictive value.

Conclusion: Doppler velocimetry might constitute an additional tool to be incorporated in clinical and ultrasonographic investigation concerning the PCOS diagnosis.

KEY WORDS: Polycystic ovary syndrome. Hirsutism. Doppler velocimetry.

\section{Referências}

1. Mather KJ, Kwan F, Corenblum B. Hyperinsulinemia in polycystic ovary syndrome correlates with increased cardiovascular risk independent of obesity. Fertil Steril 2000; 73:150-6.

2. Ehrmann DA, Barnes RB, Rosenfield RL. Polycystic ovary syndrome as a form of functional ovarian hyperandrogenism due to dysregulation of androgen secretion. Endocr Rev 1995; 16:32253.

3. Zaidi J, Campbell S, Pittrof R, et al. Ovarian stromal blood flow in women with polycystic ovaries - a possible new marker for diagnosis? Hum Reprod 1995; 10:1992-6.

4. Givens JR, Andersen RN, Umstot ES, Wiser WL. Clinical findings and hormonal responses in patients with polycystic ovarian disease with normal versus elevated LH levels. Obstet Gynecol 1976; 47:388-94.

5. Dewailly D. Definition and significance of polycystic ovaries. Baillières Clin Obstet Gynaecol 1997; 11:349-68.

6. Adams J, Frank S, Polson DW, et al. Multifollicular ovaries: clinical and endocrine features and response to pulsatile gonadotropin releasing hormone. Lancet 1985; 2:1375-9.

7. Crowley WF Jr, Hall JE, Martin KA, Adams J, Taylor AE. An overview of the diagnostic considerations in polycystic ovarian syndrome. Ann N Y Acad Sci 1993; 687:235-41. 
8. Battaglia C, Artini PG, D’Ambrogio G, Genazzani AD, Genazzani AR. The role of color Doppler imaging in the diagnosis of polycystic ovary syndrome. Am J Obstet Gynecol 1995; 172:108-13.

9. Aleem FA, Predanic M. Transvaginal color Doppler determination of the ovarian and uterine blood flow characteristics in polycystic ovary disease. Fertil Steril 1996; 65:510-6.

10.Ferriman D, Gallwey JD. Clinical assessment of body hair growth in women. J Clin Endocrinol Metab 1961; 21:1440-5.

11.Battaglia C, Artini PG, D’Ambrogio G, Galli PA, Genazzani AR. Uterine and ovarian blood flow measurement. Does the full bladder modify the flow resistance? Acta Obstet Gynecol Scand 1994; 73:716-8.

12.Zaidi J, Jurkovic D, Campbell S, Okokon E, Tan SL. Circadian variation in uterine artery blood flow indices during the follicular phase of the menstrual cycle. Ultrasound Obstet Gynecol 1995; 5:406-10.

13.Maulik D. Spectral Doppler sonography: waveform analysis and hemodynamic interpretation. In: Maulik D, editor. Doppler Ultrasound in Obstetrics \& Gynecology. 1st ed. New York: Springer-Verlag; 1997. p.43-67.

14.Tíras MB, Yalçin R, Noyan V, et al. Alterations in cardiac flow parameters in patients with polycystic ovarian syndrome. Hum Reprod 1999; 14:1949-52.
15.Vrtacnik-Bokal E, Vrtovec-Meden H. Utero-ovarian arterial blood flow and hormonal profile in patients with polycystic ovary syndrome. Hum Reprod 1998; 13:815-21.

16. Avila MAP, Murta CGV. Ovários policísticos: subsídios ultra-sonográficos. Femina 2000; 28:181-5.

17.Bruno RB, Avila MAP. Síndrome dos ovários policísticos - perfil hormonal básico. GO Atual 1999; 7:30-3.

18.Agrawal R, Sladkevicius P, Engmann L, et al. Serum vascular endothelial growth factor concentrations and ovarian stromal blood flow are increased in women with polycystic ovaries. Hum Reprod 1998; 13:651-5.

19.Engmann L, Sladkevicius P, Agrawal R, Bekir J, Campbell S, Tan SL. The pattern of changes in ovarian stromal and uterine artery blood flow velocities during in vitro fertilization treatment and its relationship with outcome of the cycle. Ultrasound Obstet Gynecol 1999; 13:26-33.

20.Battaglia C, Artini PG, Salvatori M, et al. Ultrasonographic patterns of polycystic ovaries: color Doppler and hormonal correlations. Ultrasound Obstet Gynecol 1998; 11:332-6.

21.Battaglia C, Genazzani AD, Artini PG, Salvatori M, Giulini S, Volpe A. Ultrasonographic and color Doppler analysis in the treatment of polycystic ovary syndrome. Ultrasound Obstet Gynecol 1998; 12:180-7.

\title{
COMUNICADO AOS ASSOCIADOS E LEITORES
}

\section{FEBRASGO na Internet}

Para informações e consultas temos à disposição quatro endereços eletrônicos:

\author{
febrasgopresiden@uol.com.br \\ secretaria_executiva@febrasgo.org.br \\ publicacoes@febrasgo.org.br \\ tego_habilitacao@febrasgo.org.br
}

